\title{
Contribución al conocimiento de la distribución de aves poco comunes de los talares del noreste de la provincia de Buenos Aires
}

Contribution to the knowledge of some rare birds' distribution in the Northeast Buenos Aires province

María Belén Pérez * María Agustina Caruso

División Zoología Vertebrados, Museo de La Plata, Facultad de Ciencias Naturales y Museo, Universidad Nacional de La Plata. Paseo del Bosque s/n (B1900FWA), La Plata, Argentina. E-mail: belupmaria@gmail.com; achu.caru@gmail.com

\section{RESUMEN}

En el marco de un programa de monitoreo de las aves que utilizan los talares del noreste de la provincia de Buenos Aires como sitio de nidificación, presentamos registros de aves poco comunes en este hábitat y brindamos información sobre las fechas y tipo de hábitat donde se produjeron estos registros. Nos enfocamos en especies cuya distribución no incluye regularmente a los talares del noreste bonaerense (Halconcito Gris Spiziapteryx circumcincta, Tingazú Piaya cayana, Carpintero Blanco Melanerpes candidus, Anó Chico Crotophaga ani, Loro Choclero Pionus maximiliani, Catita Chirirí Brotogeris chiriri, Atajacaminos Tijera Hydropsalis torquata y Viudita Blanca Fluvicola albiventer) y de este modo contribuir con datos de campo para una mejor comprensión de la distribución de estas especies de aves.

Palabras clave - Argentina, aves neotropicales, límite de distribución, talares bonaerenses.

\begin{abstract}
Within the framework of a bird monitoring program that reproduce in the northeast of Buenos Aires province, we present observations of rare birds in this habitat and provide information on the dates and type of habitat where the observations occurred.

Ref. bibliográfica: Pérez, M. B.; Caruso, M. A. 2020. "Contribución al conocimiento de la distribución de algunas aves poco comunes de los talares del noreste de la provincia de Buenos Aires". Acta zoológica lilloana 64 (2): 187-201. Fundación Miguel Lillo, Tucumán, Argentina. D.O.I.: https://doi.org/10.30550/ j.azl/2020.64.2/8

> Recibido: 13 de junio 2020 - Aceptado: 18 de setiembre 2020.

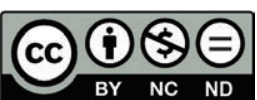


We focus on species whose distribution does not regularly include the northeastern of Buenos Aires province (Spot-winged Falconet Spiziapteryx circumcincta, Squirrel Cuckoo Piaya cayana, White Woodpecker Melanerpes candidus, Smooth-billed Ani Crotophaga ani, Scaly-headed Parrot Pionus maximiliani, Yellow-chevroned Parakeet Brotogeris chiriri, Scissor-tailed Nightjar Hydropsalis torquata and Black-backed WaterTyrant Fluvicola albiventer) and in order to contribute field data to the distribution of these birds with few records in the area.

Keywords - Argentina, distribution limit, neotropical birds, talares bonaerenses.

La información actualizada sobre la distribución de las aves en sus hábitats naturales es un importante componente para entender la dinámica de sus poblaciones y la mejor comprensión de sus historias de vida (Sullivan et al., 2017). En este sentido, estos datos toman especial relevancia a la hora de proponer acciones de manejo y conservación sobre las poblaciones de aves silvestres. En la región Neotropical, la información sobre las historias de vida de las aves en general es aún escasa, y más aún en las regiones templadas del cono sur del continente (Martin, 1996; Xiao et al., 2017). El propósito de este trabajo es aportar información sobre avistajes de algunas especies de aves con registros previos escasos en el noreste de la provincia de Buenos Aires, así como las fechas de las observaciones y los ambientes en donde estas fueron observadas.

Las observaciones se realizaron en el marco de un proyecto de monitoreo de las aves que se reproducen en bosques nativos del noreste de la provincia de Buenos Aires, el cual se desarrolla ininterrumpidamente desde el año 2005 en las Estancias 'La Matilde' ( $\left.35^{\circ} 21^{\prime} \mathrm{S}, 57^{\circ} 11^{\prime} \mathrm{O}\right)$ y 'Luis Chico' ( $\left.35^{\circ} 19^{\prime} \mathrm{S}, 57^{\circ} 11^{\prime} \mathrm{O}\right)$, partido de Punta Indio, Buenos Aires, Argentina. El ambiente natural se conoce localmente como 'talares' y es considerado un área importante para la conservación de las aves (Hummel y Rodriguez, 2005; Di Giacomo, De Francesco y Coconier, 2007). Los talares en el sitio de estudio están dominados por Tala (Celtis tala) y Coronillo (Scutia buxifolia) (Fig. 1) y ocupan las áreas de relieve positivo (Parodi 1940) constituidas por depósitos calcáreos (conchilla) (Fidalgo, Colado y De Francesco, 1973). Estos depósitos forman cordones paralelos a la costa y alternan con áreas de relieve negativo cubiertas por pastizales anegables (Arturi y Goya 2004). También en el área de estudio encontramos con frecuencia árboles exóticos, como Mora (Morus alba), Acacia Australiana (Acacia melanoxylon), Álamo (Populus spp.), Paraíso (Melia azedarach), y Acacia Negra (Gleditsia triacanthos) (Arturi y Goya 2004). La región se caracteriza por tener veranos cálidos y lluviosos, con la mayoría de las precipitaciones durante los meses de octubre y marzo. El Servicio Meteorológico Nacional Argentino reporta una precipitación anual de $1023 \mathrm{~mm}$ y una temperatura media anual de $16{ }^{\circ} \mathrm{C}$ para el período 1981-2010. La cercanía del Río de La Plata y el Océano Atlántico confieren características oceánicas al clima de esa región, generando bajas amplitudes térmicas diarias y estacionales.

Estos bosques cobran especial relevancia en términos de conservación porque son usados como sitios efectivos de alimentación y cría por numerosas especies de 


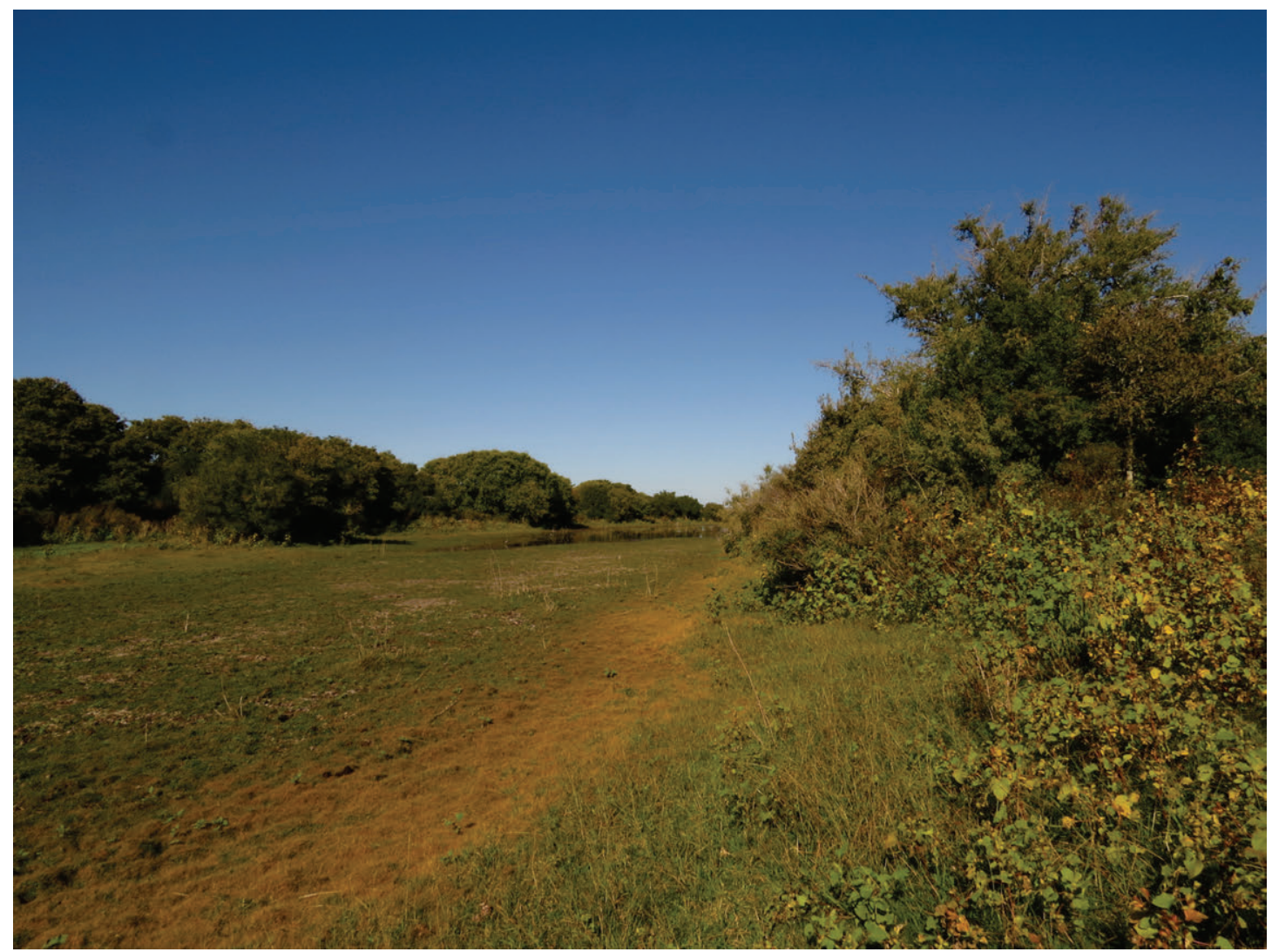

Figura 1. Imagen del bosque nativo donde realizamos las observaciones, en la Estancia La Matilde, Punta Indio, Buenos Aires.

Figura 1. Native forest's Image where we made the observations, at Estancia La Matilde, Punta Indio, Buenos Aires.

aves (Marateo, Segura y Arturi, 2009; Pagano y Merida, 2009). Dentro de los mismos, el Parque Costero del Sur ha sido considerado Área Importante para la Conservación de las Aves (AICA) (Hummel y Rodríguez 2005). En él se han registrado cinco especies de aves globalmente amenazadas y un endemismo (Espartillero Pampeano, Asthenes hudsoni) (Hummel y Rodríguez 2005). Esta área ha sufrido un proceso de degradación muy importante desde principios de siglo XX debido a la expansión de las fronteras urbana y agropecuaria, la utilización de su madera como leña, extracción de material calcáreo, ganadería, y plantaciones de bosques comerciales (Arturi y Goya, 2004).

Las observaciones no parten de un monitoreo sistemático de las poblaciones de aves en estos bosques, sino que representan una acumulación de avistajes entre el 2005 y 2019 en el marco de otros proyectos de investigación sobre aves en el área de estudio. Los avistajes se realizaron siempre con el uso de binoculares, durante la mañana (6:30 a 11:30 h) o tarde (16:00 a 19:30 h), en ausencia de lluvias o vientos intensos y fueron tomadas por investigadores y asistentes de campo que participaron del proyecto. 


\title{
LISTADO DE ESPECIES DE AVES REGISTRADAS Y DATOS RELEVANTES DE LAS OBSERVACIONES
}

\author{
Halconcito Gris \\ Spiziapteryx circumcincta (Kaup, 1852)
}

Su distribución incluye a Paraguay, este de Bolivia, norte y centro de Argentina hasta Rio Negro y una pequeña región al sur de Santa Cruz (Lopez-Lanus, 2017; Bierregaard, Kirwan y Marks, 2020). Durante las observaciones se lo vio con frecuencia sobrevolando las zonas aledañas al casco de la Estancia Luis Chico y también sobre parches de bosques y remanentes de pastizal entre los cordones boscosos (Tabla 1, Fig. 2A). Durante uno de los avistajes el individuo llevaba consigo una torcaza ( $\mathrm{Ze}$ naida auriculata) en dirección al casco de la Estancia Luis Chico (dada la fecha de la observación, presumiblemente para sus pichones, Tabla 1).

Esta especie fue avistada anteriormente en la estancia San Isidro, en el partido de Magdalena (Marateo, Rey y Glaz, 2001); en la isla Martín García (Ferrari y Henschke, 1997); en San Nicolás (Zapata y Martinez, 1972) y en Ensenada (Clerici y Aguirre, 2002) (Fig. 2A). Los registros tomados en diferentes años y estaciones (Tabla 1) indicarían que esta especie se estaría reproduciendo en el área de estudio y que no se trata de un registro ocasional. La suma de avistajes de los últimos años podría indicar la existencia de una población residente en el noreste de Buenos Aires (Pagano y Merida, 2009). Teniendo en cuenta que esta especie es frecuente en sitios con vegetación xerófila del centro y norte del país (Marateo et al., 2001) y que en el noreste de la provincia de Buenos Aires hay posibilidad de desarrollo de este tipo de vegetación (Arturi y Goya, 2004), podría inferirse que esta especie tiene las condiciones apropiadas para establecerse como residente permanente en el noreste bonaerense.

\section{Tingazú \\ Piaya cayana (Linnaeus, 1766)}

Se distribuye desde el centro de México hasta el noreste de Argentina (Payne y Kirwan, 2020). Durante las observaciones se lo vio en áreas arboladas sin intervención antrópica y siempre en invierno (Tabla 1, Fig. 2B). Esta especie ya fue avistada con anterioridad en los talares bonaerenses por Carman (1979) y Pagano y Merida (2009). Según EcoRegistros (2020), esta especie también fue observada en la Reserva Privada La Amanda en el Parque Costero del Sur en los años 2006, 2007 y 2018, en Punta Indio en el año 2013 y el 2018, en la Reserva Natural Punta Rasa en el mes de noviembre de año 2017 y en Maipú en el mes de enero de 2019 (Fig. 2B).

En los registros de e-Bird (2020), esta especie fue observada en: la Reserva Natural Punta Lara en octubre del año 1988; la Reserva Ecológica Costanera Sur en octubre de 2002; en Punta Indio en noviembre de 2013; en el 2015 en los meses de mayo, octubre y noviembre de 2015, en 2018 durante mayo y en Berisso en junio de 2018 (Fig. 2B). Debido a los recientes registros y a su aparición en distintos meses a 
Tabla 1. Detalle de las observaciones en las estancias La Matilde y Luis Chico, partido de Punta Indio, Buenos Aires. Donde $\mathrm{N}$ es el número de individuos de cada especie identificada, la fecha y observaciones sobre el tipo de hábitat y/o actividad al momento del registro.

Table 1. Detail of the observations in the Estancia La Matilde and Estancia Luis Chico, Punta Indio district, Buenos Aires. Where $\mathrm{N}$ is the number of individuals of each identified species, the date and observations on the type of habitat and / or activity at the time of registration.

\begin{tabular}{|c|c|c|c|}
\hline Especie & $\mathbf{N}$ & Fecha & Zona de observación \\
\hline \multirow[t]{9}{*}{ Spiziapteryx circumcincta } & 1 & $22 / 09 / 2005$ & Talares \\
\hline & 1 & $21 / 07 / 2006$ & Talares \\
\hline & 1 & $26 / 12 / 2008$ & Talares \\
\hline & 1 & $10 / 12 / 2009$ & Talares \\
\hline & 1 & $02 / 02 / 2015$ & Talares \\
\hline & 1 & $07 / 02 / 2015$ & Casco de la estancia Luis Chico \\
\hline & 2 & $03 / 10 / 2015$ & Casco de la estancia Luis Chico \\
\hline & 1 & $17 / 10 / 2017$ & Talares \\
\hline & 1 & $22 / 11 / 2018$ & Casco de la estancia Luis Chico \\
\hline \multirow[t]{3}{*}{ Piaya cayana } & 1 & $02 / 07 / 2006$ & Talares, cerca del río \\
\hline & 1 & $17 / 08 / 2006$ & Talares \\
\hline & 1 & $12 / 09 / 2009$ & Talares, cerca del río \\
\hline \multirow[t]{10}{*}{ Melanerpes candidus } & 5 & $26 / 09 / 2005$ & Talares \\
\hline & 6 & $02 / 12 / 2008$ & Talares \\
\hline & 3 & $14 / 12 / 2012$ & Talares \\
\hline & 5 & $14 / 02 / 2013$ & Talares \\
\hline & 5 & $17 / 02 / 2013$ & Talares \\
\hline & 5 & $07 / 09 / 2015$ & Talares \\
\hline & 3 & $16 / 10 / 2016$ & Talares \\
\hline & 7 & $23 / 10 / 2017$ & Casco de la estancia Luis Chico \\
\hline & 8 & $11 / 01 / 2018$ & Talares \\
\hline & 4 & $24 / 01 / 2018$ & Casco de la estancia Luis Chico \\
\hline Crotophaga ani & 1 & $26 / 01 / 2016$ & $\begin{array}{l}\text { Sector con abundante Coronillo, cerca del } \\
\text { casco de la Estancia Luis Chico }\end{array}$ \\
\hline \multirow[t]{7}{*}{ Pionus maximiliani } & 3 & $12 / 2 / 2016$ & Casco de la Estancia Luis Chico \\
\hline & 5 & $17 / 02 / 2016$ & Casco de la Estancia Luis Chico \\
\hline & 3 & $25 / 8 / 2017$ & Casco de la Estancia Luis Chico \\
\hline & 9 & $01 / 10 / 2017$ & Casco de la Estancia Luis Chico \\
\hline & 1 & $13 / 10 / 2017$ & Casco de la Estancia Luis Chico \\
\hline & 1 & $04 / 04 / 2018$ & Casco de la Estancia Luis Chico \\
\hline & 3 & $11 / 10 / 2018$ & Casco de la Estancia Luis Chico \\
\hline \multirow[t]{2}{*}{ Brotogeris chiriri } & 2 & $29 / 12 / 2016$ & Casco de la Estancia Luis Chico \\
\hline & 3 & $12 / 02 / 2017$ & Casco de la Estancia Luis Chico \\
\hline \multirow[t]{4}{*}{ Hydropsalis torquata } & 1 & $24 / 12 / 2008$ & En el suelo, entre los cordones de talas \\
\hline & 1 & $01 / 11 / 2011$ & En el suelo, entre los cordones de talas \\
\hline & 1 & $13 / 12 / 2016$ & $\begin{array}{l}\text { Posado sobre una rama, a baja altura, } \\
\text { entre los cordones de talas }\end{array}$ \\
\hline & 1 & $16 / 01 / 2017$ & En el suelo, entre los cordones de talas \\
\hline \multirow[t]{5}{*}{ Fluvicola albiventer } & 2 & $13 / 12 / 2017$ & $\begin{array}{l}\text { Laguna entre los cordones de tala. } \\
\text { Nidificando }\end{array}$ \\
\hline & 2 & $01 / 02 / 2018$ & $\begin{array}{l}\text { Laguna entre los cordones de tala. } \\
\text { Nidificando }\end{array}$ \\
\hline & 1 & $20 / 02 / 2018$ & $\begin{array}{l}\text { Laguna entre los cordones de tala. } \\
\text { Nidificando }\end{array}$ \\
\hline & 1 & $26 / 11 / 2018$ & $\begin{array}{l}\text { Laguna entre los cordones de tala. } \\
\text { Nidificando }\end{array}$ \\
\hline & 2 & $11 / 01 / 2019$ & $\begin{array}{l}\text { Laguna entre los cordones de tala. } \\
\text { Nidificando }\end{array}$ \\
\hline
\end{tabular}


lo largo del año, se sugiere incluir el noreste de la provincia de Buenos Aires como parte de su distribución natural.

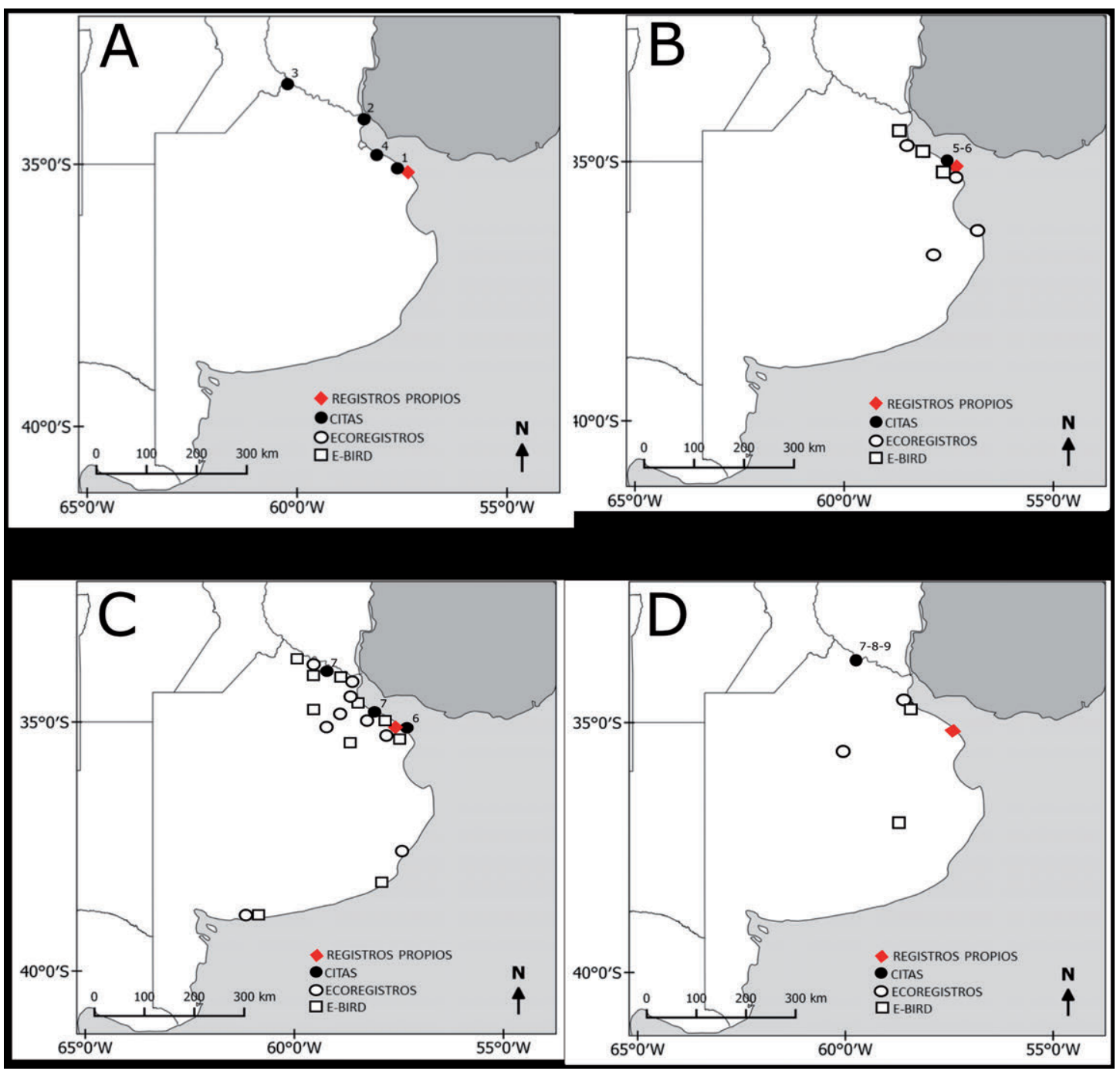

Figura 2. Mapas de la provincia de Buenos Aires con la ubicación de nuestras observaciones (rombo rojo), de otros registros ya publicados (círculo negro) y los avistajes disponibles en plataformas web como EcoRegistros (círculo blanco) y e-Bird (cuadrado blanco) para el Halconcito Gris Spiziapteryx circumcincta (A), Tingazú Piaya cayana (B), Carpintero Blanco Melanerpes candidus (C) y Anó Chico Crotophaga ani (D). Los números sobre los círculos negros indican la fuente: [1] Marateo et al. (2001), [2] Ferrari y Henschke (1997), [3] Zapata y Martinez (1972), [4] Clerici y Aguirre (2002), [5] Carman (1979), [6] Pagano y Merida (2009), [7] Bodrati (2001), [8] Bodrati et al. (2006) y [9] Sierra (2006).

Figure 2. Maps of the province of Buenos Aires with the location of our observations (red diamond), other records already published (black circle) and the sightings available on web platforms such as EcoRegistros (white circle) and e-Bird (white square) for the Spot-winged Falconet Spiziapteryx circumcincta (A), Squirrel Cuckoo Piaya cayana (B), White Woodpecker Melanerpes candidus (C) and Smooth-billed Ani Crotophaga ani (D). The numbers above the black circles indicate the source: [1] Marateo et al. (2001), [2] Ferrari and Henschke (1997), [3] Zapata and Martinez (1972), [4] Clerici and Aguirre (2002), [5] Carman (1979), [6] Pagano and Merida (2009 ), [7] Bodrati (2001), [8] Bodrati et al. (2006) and [9] Sierra (2006). 


\section{Carpintero Blanco \\ Melanerpes candidus (Otto, 1796)}

Se distribuye desde el extremo sur de Perú, centro y noreste de Brasil, Paraguay, Bolivia, Uruguay hasta el centro de Argentina, incluyendo el norte de la provincia de Buenos Aires (Winkler, Christie y Kirwan, 2020). Los ejemplares siempre fueron observados moviéndose en grupos de entre tres y ocho individuos, en distintos momentos del año (Tabla 1). En uno de los avistajes se observó a uno de los individuos del grupo construyendo una cavidad, presumiblemente para usarla como nido. Esta especie ha sido registrada con anterioridad en inmediaciones del Parque Costero del Sur (Pagano y Merida, 2009) y en distintos sitios dentro del territorio bonaerense (Fig. 2C). Si bien en la provincia de Buenos Aires se la consideró una especie "hipotética" en función de dos registros antiguos (Narosky y Di Giacomo, 1993; Celsi, Milat y Kildegaard, 2014), en la actualidad hay suficientes registros (e-Bird, 2020; EcoRegistros, 2020) como para considerarla residente permanente en los talares del noreste de la provincia de Buenos Aires.

\section{Anó Chico \\ Crotophaga ani (Linnaeus, 1758)}

Se distribuye desde el extremo sur de México y sureste de Estados Unidos, pasando por islas del Caribe, hasta Uruguay y el centro-norte de Argentina (Payne y Kirwan, 2020). Esta especie fue avistada por única vez en un área boscosa con dominio de Coronillos (árbol nativo) y Acacia Negra (árbol exótico), en las inmediaciones del casco de la estancia Luis Chico (Tabla 1, Fig. 2D). Tanto para Narosky y Di Giacomo (1993) como para Darrieu y Camperi (2001), la especie presenta escasos registros en el noreste de la provincia de Buenos Aires. En los talares de San Pedro y Vuelta de Obligado, la presencia de esta especie es ocasional (Bodrati, 2001; Bodrati, Mérida, Bodrati y Sierra, 2006; Sierra, 2006) y no presenta un claro patrón migratorio en el área. Según EcoRegistros (2020), existen solo tres avistajes en la provincia de Buenos Aires (Fig. 2D): uno en Saladillo en noviembre de 2006 y los otros dos en la Reserva Ecológica Costanera Sur en diciembre del mismo año. E-Bird (2020) también menciona solamente tres registros: dos en la Reserva Ecológica Costanera Sur en diciembre de 1985 y 2016 y uno en Tandil en mayo de 2016 (Fig. 2D). Debido a que solo lo observamos una única vez en nuestro sitio de estudio y que en la provincia de Buenos Aires hay pocos registros, no descartamos que el individuo visto provenga de la liberación de ejemplares en cautiverio.

\section{Loro Choclero \\ Pionus maximiliani (Kuhl, 1820)}

Su distribución abarca Paraguay, sureste de Bolivia, este y sur de Brasil y norte de Argentina (Collar, Bonan y Boesman, 2020). Los avistajes ubican a esta especie vo- 


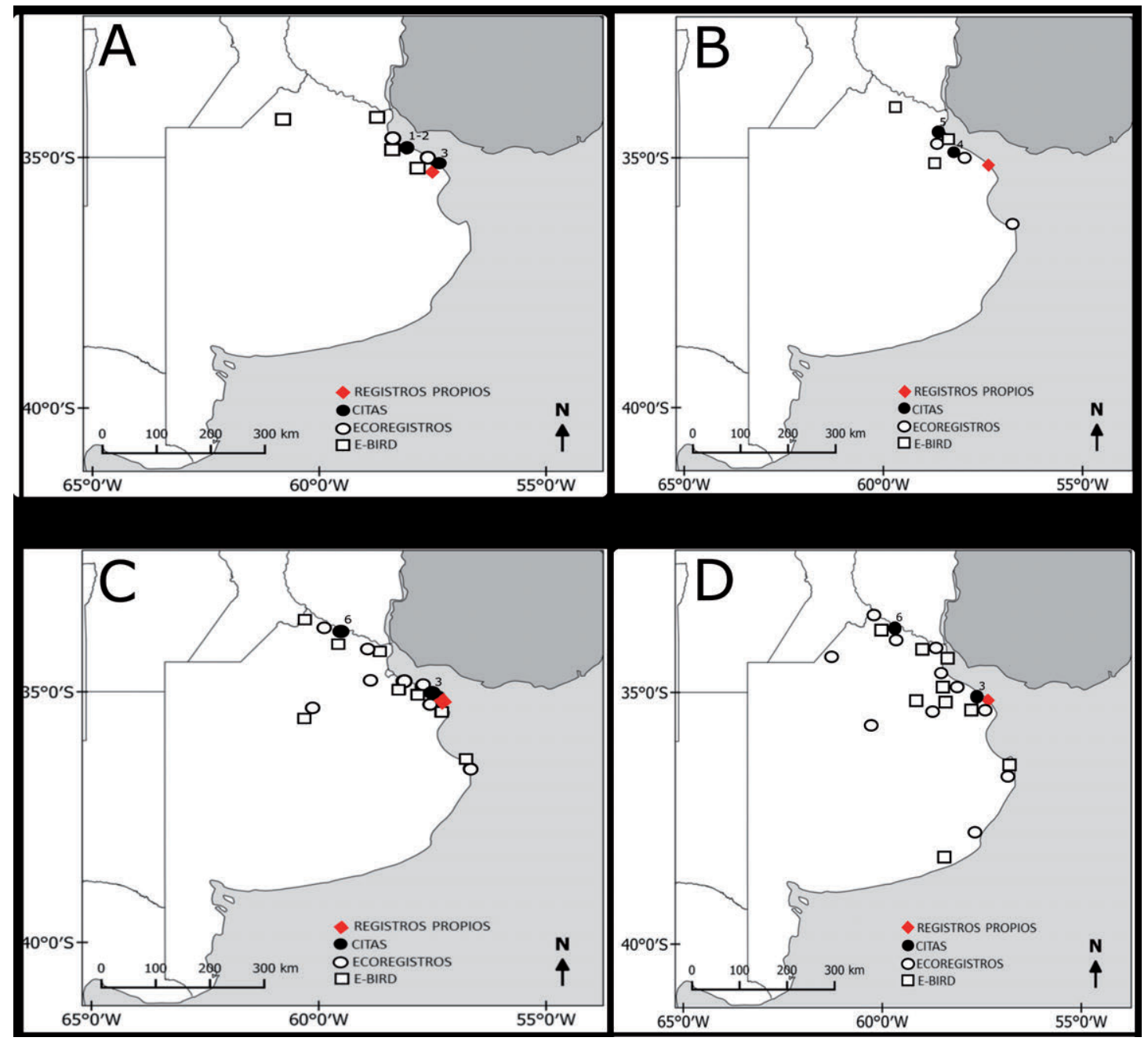

Figura 3. Mapas de la provincia de Buenos Aires con la ubicación de nuestras observaciones (rombo rojo), de otros registros ya publicados (círculo negro) y los avistajes disponibles en plataformas web como EcoRegistros (círculo blanco) y e-Bird (cuadrado blanco) para el Loro Choclero Pionus maximiliani (A), Catita Chirirí Brotogeris chiriri (B), Atajacaminos Tijera Hydropsalis torquata (C) y Viudita Blanca Fluvicola albiventer (D). Los números sobre los círculos negros indican la fuente: [1] Agnolin et al. (2009), [2] Ibáñez et al. (2014), [3] Pagano y Merida (2009), [4] Pérez (1990), [5] Gasparri et al. (2018) y [6] Bodrati et al. (2006).

Figure 3. Maps of the province of Buenos Aires with the location of our observations (red diamond), of other records already published (black circle) and the sightings available on web platforms such as EcoRegistros (white circle) and e-Bird (white square) for the Scaly-headed Parrot Pionus maximiliani (A), Yellow-chevroned Parakeet Brotogeris chiriri (B), Scissor-tailed Nightjar Hydropsalis torquata (C) and Black-backed Water-Tyrant Fluvicola albiventer (D). The numbers above the solid circles indicate the source: [1] Agnolin et al. (2009), [2] Ibáñez et al. (2014), [3] Pagano and Merida (2009), [4] Pérez (1990), [5] Gasparri et al. (2018) and [6] Bodrati et al. (2006).

lando en grupos de variado tamaño (Tabla 1), siempre en las inmediaciones del casco de la Estancia Luis Chico (Fig. 3A). Hace una década, esta especie era observada frecuentemente en la provincia de Buenos Aires, aunque generalmente se trataba de ejemplares liberados del cautiverio y no formaban grandes bandadas (Haene, 2006). 
En julio del año 2000, Agnolin, Chimento, Frank y Lucero (2009) observaron bandadas en la estancia Abril (ubicada entre las ciudades de Florencio Varela y La Plata). También han sido observadas bandadas y parejas reproductoras en el Parque Provincial Pereyra Iraola (Ibañez, Girini, Palacio y Montalti, 2014). Scheffer, Cremasch, Montalti y Grilli (2015) clasifican a esta especie como "común” en el Parque Provincial Pereyra Iraola. Además se observaron ocho ejemplares en Punta Indio en marzo del año 2007 y 2009 (Pagano y Merida, 2009). En EcoRegistros (2020) hay avistajes en el norte (La Plata, Berazategui y Ciudad Autónoma de Buenos Aires) y noreste (Punta Indio y Magdalena) de la provincia de Buenos Aires. A su vez en e-Bird (2020) también figuran avistajes en Punta Indio, La Plata, Berazategui y Ciudad Autónoma de Buenos Aires. El origen de estas poblaciones, aparentemente en expansión dentro de la provincia de Buenos Aires, probablemente se asocie con sueltas de individuos provenientes del cautiverio, ya que las poblaciones de Loro Choclero más cercanas se encuentran a más de mil kilómetros en el norte de Argentina. Esta especie ha logrado adaptarse satisfactoriamente a este nuevo ambiente y reproducirse en las cavidades de árboles (Ibañez et al., 2014), lo que nos lleva a considerarla 'común' en el noreste de la provincia de Buenos Aires.

\section{Catita Chirirí \\ Brotogeris chiriri (Vieillot, 1818)}

Se distribuye desde el norte de Bolivia, Paraguay, centro y sureste de Brasil y noreste de Argentina (Collar et al., 2020). Durante las observaciones se los vio en pequeños grupos durante la temporada estival (Tabla 1). Los primeros avistajes en la zona centro-este del país fueron en 1988 y 1989 en la Ciudad Autónoma de Buenos Aires (Pérez, 1990; Fernández, 1991). Un trabajo más reciente indica que actualmente se la considera una especie asilvestrada (que vive y se reproduce de forma natural y espontánea pese a estar introducida en un lugar ajeno a su área original), común y residente en la zona de San Isidro, provincia de Buenos Aires (Gasparri et al., 2018). Otros autores (Navas y Bó, 1996; Blendinger, 1998; Burgos-Gallardo y Grilli, 2018) también presentan registros en el norte y noreste de la provincia de Buenos Aires, aunque Scheffer et al. (2015) mencionan que los avistajes en la zona del Parque Pereyra Iraola son inusuales. Lopez-Lanuz (2017) lo cita al noreste de la provincia de Buenos Aires como especie introducida; e-Bird (2020) y EcoRegistros (2020) (Fig. 3B) muestran numerosos registros actuales para la Ciudad Autónoma de Buenos Aires y ciudades del conurbano bonaerense (Fig. 3B), por lo que podría considerarse que estas poblaciones están en expansión.

\section{Atajacaminos Tijera \\ Hydropsalis torquata (Gmelin, 1789)}

Su distribución incluye Perú, Brasil, Bolivia, Paraguay, Uruguay y Argentina, donde se extiende desde el norte al centro, hasta el norte de Neuquén y Río Negro 
(Bianchini, M., 2016; Cleere, 2020). Las observaciones realizadas lo sitúan entre los cordones de Talares (Fig. 3C), posado sobre ramas a baja altura o en el suelo y rodeado de abundante excremento seco de ganado vacuno. Según Pagano y Mérida (2009) y Bodrati et al. (2006) es un visitante estival en áreas abiertas y talares del norte bonaerense y posiblemente nidifica en la zona. En plataformas como e-Bird (2020) y EcoRegistros (2020) se reportan algunos pocos avistajes en Berisso, Magdalena, Punta Indio y San Clemente. Los escasos registros probablemente se deban a su comportamiento solitario y silencioso mientras se encuentra en reposo, lo que dificulta su hallazgo.

\section{Viudita Blanca \\ Fluvicola albiventer (Spix, 1825)}

Se distribuye desde el este de Perú, Brasil, Bolivia, Paraguay, oeste de Uruguay y noreste de Argentina (Farnsworth y Langham, 2020). Las poblaciones del sur de su área de distribución migran más al norte luego de su etapa reproductiva (Farnsworth y Langham, 2020). A pesar de recorrer frecuentemente bordes de lagunas con cordones de Talares desde el 2005, recién en 2017 observamos por primera vez a esta especie en el sitio de estudio, en todas las ocasiones cerca de su conspicuo nido. Pagano y Mérida (2009) y Bodrati et al. (2006) la mencionan como posible nidificante, aunque escaso, en la zona del Parque Costero del Sur. En plataformas como e-Bird (2020) y EcoRegistros (2020), existen algunas pocas observaciones en el noreste de Buenos Aires, pero abundantes registros para el norte y noroeste de la provincia (Fig. 3D). La falta de registros en nuestro sitio de estudio entre 2005 y 2017 podría deberse a que esta especie tendría un comportamiento oportunista en relación a los cuerpos de agua que se forman entre los cordones de Talares.

\section{CONSIDERACIONES FINALES}

Con estos registros intentamos colaborar con el conocimiento de la distribución de algunas aves poco comunes que habitan los Talares bonaerenses. Hicimos hincapié en un pequeño grupo de aves (provenientes de distintas familias y órdenes) que habitan el cono sur de la región Neotropical, cuyas distribuciones tradicionales no las sitúan regularmente en los talares del noreste de la provincia de Buenos Aires. $\mathrm{Si}$ bien los talares en esta región son ambientes que presentan un alto grado de disturbio debido a la actividad antrópica, los remanentes de bosques en nuestro sitio de estudio se encuentran relativamente bien conservados, con especies arbóreas nativas como dominantes de la masa forestal (Segura, 2011). Esta situación podría resultar ventajosa para muchas especies de aves que usan estos bosques como sitios de nidificación (Marateo et al., 2009; Pagano y Merida, 2009; Segura y Berkunsky, 2012; Segura, Mahler, Berkunsky y Reboreda., 2015; Gonzalez, Jauregui y Segura, 2019; Jauregui, Gonzalez y Segura, 2019), posiblemente incluidas las especies tratadas en este trabajo. Dado que el área de estudio representa una región con moderada alte- 
ración antrópica (es decir, con actividad ganadera, pero sin importantes desmontes en las últimas décadas y en donde la masa forestal aún está dominada por árboles nativos) y con el objetivo de poder comprender mejor la tolerancia de estas aves a la alteración del habitat, sería interesante realizar nuevos estudios en talares con mayor grado de alteración antrópica, como por ejemplo los escasos relictos de talares continentales que aún sobreviven en zonas aledañas a Chascomús, General Belgrano o el partido de la Costa, o los aún más escasos relictos de bosque que se encuentran en zonas muy próximas al conurbano bonaerense.

Esta contribución, junto a otros estudios en aves que habitan los talares del noreste de la provincia de Buenos Aires (Marateo et al., 2009; Pagano y Merida, 2009; Pagano, Ornstein, Di Sallo y Oscar, 2017; entre otros), ofrece información sobre posibles nuevas áreas de distribución de las aves mencionadas que nos permite una mejor comprensión de sus historias de vida.

\section{AGRADECIMIENTOS}

Agradecemos a Luis Del Sotto, Emiliano Torres y María L. Shaw por permitirnos el ingreso a sus propiedades. Agradecemos especialmente a Luciano N. Segura, Adrián Jauregui, Exequiel Gonzalez, Martín Colombo y un gran número de asistentes de campo que participaron de los trabajos de campo entre 2005 y 2019.

\section{FINANCIAMIENTO}

La Universidad Nacional de La Plata (Proyectos I+D 11/N708 y 16/N833) y la Agencia Nacional de Promoción Científica y Tecnológica (PICT 2014-3347) aportaron los fondos permitieron los muestreos.

\section{PARTICIPACIÓN}

María Belén Pérez y María Agustina Caruso concibieron el estudio, organizaron las planillas con datos de campo, diseñaron los mapas y redactaron el manuscrito.

\section{CONFLICTOS DE INTERÉS}

No existen conflictos de interés entre los autores ni con terceros.

\section{BIBLIOGRAFÍA CITADA}

Agnolin, F.L., Chimento, N.R., Frank, C., Lucero, R.F. (2009). Nuevos registros de aves argentinas. Nótulas Faunísticas, 34: 1-4. 
Arturi, M.F., Goya, J.F. (2004). Estructura, dinámica y manejo de los talares del NE de Buenos Aires. M.F. Arturi, M.F., Frangi, J. \& J.F. Goya (eds.), Ecología y manejo de los bosques de Argentina. Editorial de la Universidad Nacional de La Plata, La Plata, pp. 1-23.

Bianchini, M. (2016). Registros y comportamiento del Atajacaminos Tijera ( Hydropsalis torquata, Aves: Caprimulgidae) en la Patagonia, Argentina. Acta Zoológica Lilloana, 60(2), 170-176.

Bierregaard, R.O., Kirwan Jr., G.M., Marks, J.S. (2020). Spot-winged Falconet (Spiziapteryx circumcincta)". J. del Hoyo, A. Elliott, J. Sargatal, D.A. Christie \& E. de Juana (eds.), Handbook of the Birds of the World Alive. Lynx Edicions, Barcelona (https://www.hbw.com/node/53204, acceso 5 de marzo de 2020).

Blendinger, P.G. (1998). Registros de aves poco frecuentes en la Argentina y sector Antártico Argentino. Nuestras Aves, 38: 5-8.

Bodrati, A. (2001). Notas sobre aves infrecuentes o poco conocidas para la provincia de Buenos Aires, Argentina. Nuestras Aves 41: 13-17.

Bodrati, A., Mérida, E., Bodrati, G., Sierra, E. (2006). Avifauna del talar de Vuelta de Obligado y de sus ambientes contiguos, San Pedro, provincia de Buenos Aires, Argentina. E. Mérida \& J. Athor (eds.), Talares bonaerenses y su conservación, Buenos Aires. Fundación de Historia Natural Félix de Azara, Buenos Aires, pp. 117-124,

Burgos-Gallardo, F., Grilli, P.G. (2018). Distribución de la Catita Chirirí (Brotogeris chiriri) y primer registro de nidificación en Argentina. Nuestras Aves, 63: $38-43$.

Carman, R.L. (1979). Piaya cayana en Buenos Aires. Hornero, 12: 54.

Celsi, C.C., Milat, J.A., Kildegaard, M. (2014). Hallazgo de un ejemplar de Carpintero Blanco (Melanerpes candidus) en libertad, en la costa bonaerense. Historia Natural, 4: 89-94.

Cleere, N. (2020). Scissor-tailed Nightjar (Hydropsalis torquata). J. del Hoyo, A. Elliott, J. Sargatal, D.A. Christie \& E. de Juana (eds.), Handbook of the Birds of the World Alive. Lynx Edicions, Barcelona (https://www.hbw.com/node/53204, acceso 5 de marzo de 2020).

Clerici, G.A., Aguirre, P.H. (2002). Nuevo hallazgo del halconcito gris (Spiziapteryx circumcinctus) en el nordeste de la provincia de Buenos Aires, Argentina. Nuestras Aves, 43: 26.

Collar, N., Bonan, A., Boesman, P. (2020). Scaly-headed Parrot (Pionus maximiliani). J. del Hoyo, A. Elliott, J. Sargatal, D.A. Christie \& E. de Juana (eds.), Handbook of the Birds of the World Alive. Lynx Edicions, Barcelona (https://www.hbw. com/node/53204, acceso 5 de marzo de 2020).

Darrieu, C.A., Camperi, A. (2001). Nueva lista de las aves de la provincia de Buenos Aires, monografía, Universidad Nacional de La Plata, La Plata.

Di Giacomo, A.S., De Francesco, V., Coconier, E.G. (2007). Áreas de Importancia para la Conservación de las Aves en la Argentina. Aves Argentinas/Asociación Ornitológica del Plata, Buenos Aires.

eBird. (2020). eBird: an Online Database of Bird Distribution and Abundance (http://www.ebird.org, acceso 20 marzo de 2020). 
EcoRegistros. (2020). EcoRegistros: Distribution map (http://www.ecoregistros.org, acceso 20 de marzo de 2020).

Farnsworth, A., Langham, G. (2020). Black-backed Water-tyrant (Fluvicola albiventer). J. del Hoyo, A. Elliott, J. Sargatal, D.A. Christie \& E. de Juana (eds.), Handbook of the Birds of the World Alive. Lynx Edicions, Barcelona (https:// www.hbw.com/node/53204, acceso 5 de marzo de 2020).

Fernández H.G. (1991). Psitácidos asilvestrados en el partido de San Isidro (Prov. de Buenos Aires). Garganchillo 11:12-13

Ferrari, C., Henschke, C. (1997). El halconcito gris (Spiziapteryx circumcinctus) en la isla Martín García, Provincia de Buenos Aires, Argentina. Nuestras Aves, $36: 5$.

Fidalgo F., Colado O., De Francesco F.O. (1973). Sobre ingresiones marinas en los partidos de Castelli, Chascomús y Magdalena (Provincia de Buenos Aires). Actas del V Congreso Geológico. Argentino, 3:227-247.

Gasparri, B., Del Río, D., Earnshaw, A., Henschke, C., Bryant, G., Cristaldo, L., Faccioli, A., Gentiles, J.M., Galiano-Guirao, I., Vitale, M., Rodríguez-Tourón, G., Suárez, G. (2018). Lista comentada de las aves del Parque Natural Municipal Ribera Norte, San Isidro, Provincia de Buenos Aires, Argentina. Nótulas Faunísticas, 234: 1-43.

Gonzalez, E., Jauregui, A., Segura, L. N. (2019). Breeding biology of the Yellowbrowed Tyrant (Satrapa icterophrys) in south temperate forests of central Argentina. Wilson Journal of Ornithology, 131: 534-542.

Jauregui, A., Gonzalez, E., Segura, L. N. (2019). Nesting biology of the Narrowbilled Woodcreeper (Lepidocolaptes angustirostris) in a southern temperate forest of central-east Argentina. Studies on Neotropical Fauna and Environment, 54: 114-120.

Haene, E. (2006). Invasión de loros en la ciudad. Naturaleza y Conservación, 19: $16-23$.

Hummel, A.E., Rodríguez, E.A. (2005). Parque Costero del Sur. A. S. Di Giacomo (ed.) Áreas importantes para la conservación de las aves en la Argentina. Sitios prioritarios para la conservación de la biodiversidad, Buenos Aires. Aves Argentinas/Asociación Ornitológica del Plata, Buenos Aires, pp. 44-46,

Ibañez, L.M., Girini, J.M., Palacio, F.X., Montalti, D. (2014). Nidificación y alimentación de psittácidos introducidos en el noreste de la provincia de Buenos Aires, Argentina. Hornero, 29: 13-22.

López Lanús, B. (2017). Guía Audiornis de las Aves de Argentina: Fotos y Sonidos, Identificación por características contrapuestas y marcas sobre imágenes. Segunda edición ampliada. Buenos Aires, Argentina: Copyright Audiornis Producciones.

Marateo G., Rey, P., Glaz, D. (2001). Nuevo registro del Halconcito Gris (Spiziapteryx circumcinctus) para el nordeste de la provincia de Buenos Aires, Argentina. Nuestras aves, 41: 10-11.

Marateo, G., Segura, L.N., Arturi, M.F. (2009). Las relaciones entre las aves y el bosque en el Parque Costero del Sur. J. Athor (ed.), Parque Costero del Sur: Naturaleza, conservación y patrimonio cultural. Fundación de Historia Natural Félix de Azara, Buenos Aires, pp. 122-137 
Martin, T.E. (1996). Life history evolution in tropical and south temperate birds: what do we really know? Journal of Avian Biology, 27: 263-272.

Narosky, T. \& A. Di Giacomo. (1993). Las Aves de la provincia de Buenos Aires, distribución y estatus, Buenos Aires. Asociación Ornitológica del Plata. Editorial Vazquez Mazzini y Literature of Latin America (L.O.L.A), Buenos Aires.

Navas, J.R., N.A. Bó. (1996). Distribución geográfica y situación actual de Brotogeris versicolorus en la Argentina. Hornero, 14: 90-92.

Pagano, L.G., Mérida, E. (2009). Aves del Parque costero del Sur. J. Athor (ed.), Parque Costero del Sur: Naturaleza, conservación y patrimonio cultural. Fundación de Historia Natural Félix de Azara, Buenos Aires, pp. 200-244

Pagano, L.G., Ornstein, U., Di Sallo, F.G., Oscar, D.E. (2017). Adiciones y comentarios sobre las aves del Parque Costero del Sur, Buenos Aires, Argentina. Nuestras Aves, 62: 17-23.

Parodi L. 1940. Distribución Geográfica de los Talares la Provincia de Buenos Aires. Darwiniana, 4:33- 56.

Payne, R., Kirwan, G.M. (2020). Common Squirrel-cuckoo (Piaya cayana). J. del Hoyo, A. Elliott, J. Sargatal, D.A. Christie \& E. de Juana (eds.), Handbook of the Birds of the World Alive. Lynx Edicions, Barcelona (https://www.hbw. com/node/53204, acceso 5 de marzo de 2020).

Pérez, J.H. (1990). Catita chirirí (Brotogeris versicolurus) en Capital Federal. Nuestras Aves, 21: 27-28.

Scheffer, M., Cremaschi, L., Montalti, D., Grilli, P.G. (2015). Los loros exóticos del Parque provincial Pereyra Iraola y comentarios sobre su presencia en la ribera platense. Nuestras Aves, 60: 3-8.

Segura, L.N. (2011). Biología reproductiva del Cardenal Común (Paroaria coronata, Thraupidae) en talares del noreste de la provincia de Buenos Aires. Tesis Doctoral, Universidad Nacional de La Plata, La Plata.

Segura, L.N., Berkunsky, I. (2012). Supervivencia de nidos del Cardenal Común (Paroaria coronata) en un hábitat modificado en Argentina. Ornitologia Neotropical, 23: 489-498.

Segura, L.N., Mahler, B., Berkunsky, I., Reboreda, JC. (2015). Nesting biology of the Red-crested Cardinal (Paroaria coronata) in south temperate forests of central Argentina. Wilson Journal of Ornithology, 127: 249-258.

Sierra, E. (2006). Importancia y consolidación del Refugio Histórico Natural Vuelta de Obligado, San Pedro, provincia de Buenos Aires. E. Merida \& J. Athor (eds.), Talares bonaerenses y su conservación. Fundación de Historia Natural Félix de Azara, Buenos Aires, pp. 117-124.

Sullivan, B.L., Phillips, T., Dayer, A.A., Wood, C.L., Farnsworth, A., Iliff, M.J., Davies, I. J., Wiggins, A., Fink, D., Hochachka, W. M., Rodewald, A.D., Rosenberg, K.V., Bonney, R., Kelling, S. (2017). Using open access observational data for conservation action: A case study for birds. Biological Conservation, 208: 5-14.

Winkler, H., Christie, D.A., Kirwan, G.M. (2020). White Woodpecker (Melanerpes candidus). J. del Hoyo, A. Elliott, J. Sargatal, D.A. Christie \& E. de Juana (eds.), 
Handbook of the Birds of the World Alive. Lynx Edicions, Barcelona (https:// www.hbw.com/node/53204, acceso 5 de marzo de 2020).

Xiao, H., Hu, Y., Lang, Z., Bohao, F., Guo, W., Zhang, Q., Pan, X., Lu, X. (2017). How much do we know about the breeding biology of bird species in the world? Journal of Avian Biology, 48: 513-518.

Zapata, A.R.P., Martínez, H. S. (1972). Algunas aves no citadas y otras poco frecuentes para el sur de la provincia de Buenos Aires. Acta Zoológica Lilloana, 29: 181-199. 